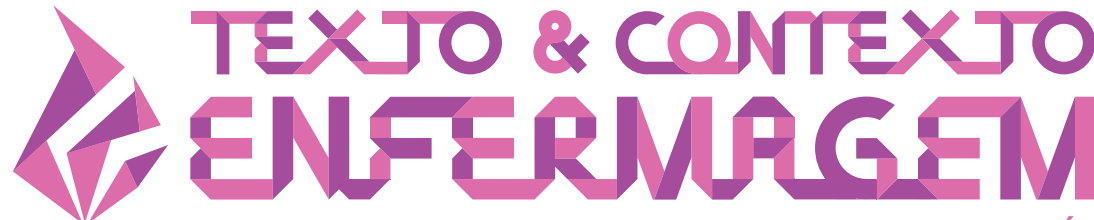

TEXT \& CONTEXT NURSING TEXTO \& CONTEXTO ENFERMERÍA

\section{TRANSCULTURAL TRANSLATION AND ADAPTATION OF CONDOM EMBARRASSMENT SCALE FOR THE BRAZILIAN CONTEXT}

\author{
Berenice Moreira $^{1}$ (D) \\ Umbelina do Rego Leite ${ }^{2}$ (B) \\ Sidclay Bezerra de Souza ${ }^{3}$ (D) \\ Marcos Pascoal Pattussi ${ }^{4}$ (D)
}

\begin{abstract}
${ }^{1}$ Universidade de Rio Verde, Faculdade de Enfermagem. Rio Verde, Goiás, Brasil. 2Universidade Federal de Pernambuco, Centro de Filosofia e Ciências Humanas. Pernambuco, Recife, Brasil. ${ }^{3}$ Universidad Católica del Maule, Facultad de Ciencias de la Salud, Departamento de Psicología. Talca, Maule, Chile. ${ }^{4}$ Universidade do Vale do Rio dos Sinos, Programa de Pós-Graduação em Saúde Coletiva. São Leopoldo, Rio Grande do Sul, Brasil.
\end{abstract}

DESCRIPTORS: Instrument adaptation studies. Scales. Cross-cultural comparison. Condoms.

Sexually transmitted diseases. 


\section{TRADUÇÃO E ADAPTAÇÃO TRANSCULTURAL DA CONDOM EMBARRASSMENT SCALE PARA O CONTEXTO BRASILEIRO}

\section{RESUMO}

Objetivo: descrever o processo de adaptação transcultural da Condom Embarrassment Scale (Escala de Constrangimento no uso do Preservativo) para utilização no contexto brasileiro.

Método: estudo metodológico que compreendeu as seguintes etapas: tradução inicial da escala original por dois tradutores independentes, síntese das traduções, tradução reversa, avaliação da tradução reversa pela autora da escala original, adaptações na versão em português por comitê de especialistas e realização do pré-teste. Tais etapas permitiram realizar as adaptações necessárias à realidade sociocultural e ao nível de compreensão da população-alvo. Participaram do pré-teste 42 universitários com idades entre 18 e 28, de ambos os sexos, de uma universidade localizada na Região Centro-Oeste do Brasil.

Resultados: levando-se em consideração as variações idiomáticas e culturais avaliadas, a versão em português demonstrou equivalência com a versão Norte-Americana.

Conclusão: a versão da escala no português do Brasil demonstrou compreensibilidade quando aplicada em universitários. Novos estudos avaliando as propriedades psicométricas da escala são necessários.

DESCRITORES: Estudo de adaptação de instrumentos. Escalas. Comparação transcultural. Preservativos. Doenças sexualmente transmissíveis.

\section{TRADUCCIÓN TRANSCULTURAL Y ADAPTACIÓN DE LA CONDOM EMBARRASSMENT SCALE PARA EL CONTEXTO BRASILEÑO}

\section{RESUMEN}

Objetivo: describir el proceso de adaptación transcultural de la Condom Embarrassment Scale (Escala de restricciones en el uso de condones) para su uso en el contexto brasileño.

Método: estudio metodológico que comprendió los siguientes pasos: traducción inicial de la escala original por dos traductores independientes, síntesis de traducciones, traducción inversa, evaluación de la traducción inversa por el autor de la escala original, adaptaciones en la versión en portugués por un comité de expertos y realización del pretest. Estos pasos han permitido realizar las adaptaciones necesarias a la realidad sociocultural y al nivel de comprensión de la población-objetivo. Participaron del pretest 42 estudiantes universitarios de ambos sexos, de 18 a 28 años, de una universidad situada en la región del medio oeste del Brasil.

Resultados: teniendo en cuenta las variaciones idiomáticas y culturales evaluadas, la versión en portugués demostró equivalencia con la versión norte americana.

Conclusión: la versión en portugués brasileño de la escala demostró ser comprensible cuando se aplicó a estudiantes universitarios. Se necesitan más estudios que evalúen las propiedades psicométricas de la escala.

DESCRIPTORES: Estudio de adaptación de instrumentos. Escalas. Comparación transcultural. Condones. Enfermedades sexualmente transmisibles. 


\section{INTRODUCTION}

Sexually transmitted infections (STIs) are a group of infections that can be acquired through unprotected sex. ${ }^{1}$ This represents a major public health challenge worldwide, especially in developing countries whose growing socio-cultural and economic contexts have a major influence on the occurrence of these infections. ${ }^{2}$

Each year, there are about 357 million new cases of curable STIs in the world, affecting both sexes, mainly in the ages between 15 and 49 years, and most of them occur in developing countries. ${ }^{3}$ In addition, STIs increase the risk of transmission of the Human Immunodeficiency Virus (HIV) and Acquired Immunodeficiency Syndrome (AIDS). Approximately 36.7 million people are living with HIV/ AIDS in the world. ${ }^{4}$

Specifically in Brazil, the detection rate for AIDS cases per inhabitant doubled from 2007 to 2017 in the 15 to 24 age group. Among young people aged 15 to 19, it went from 3.0 in 2007 to 7.0 cases/100,000 inhabitants in 2017, and among those aged 20 to 24, it went from 15.6 in 2007 to 36.2 in $2017 .^{5}$

As teenagers and young adults are a heterogeneous group with regard to experiences, social practices and lifestyle are different in their biological, mental and social development. ${ }^{6}$ They have specific needs in the areas of disease prevention and health promotion that can be reached by health policies focused on the development of critical thinking and decision making in the face of their vulnerabilities.

Upon entering university, young people will experience greater social and economic freedom and adopt new lifestyles. ${ }^{7-8}$ It is at this stage that a greater number of interpersonal relationships is established and a greater likelihood of sexual intercourse, especially without the use of condoms, in addition to being more exposed to alcohol and other legal and illegal drugs. ${ }^{9}$

Consistent condom use in all sexual relations has been considered an effective method to prevent STIs. ${ }^{10}$ Thus, instruments that assess the use of condoms have been developed in order to gain a better understanding of this contraception and STI prevention method. ${ }^{11}$

The feeling of embarrassment when purchasing the condom, negotiating its use with the sexual partner and in the use itself are the main factors that influence its non-use. In this sense, with the objective of evaluating the embarrassment in relation to the use of condoms when purchasing, negotiating the use sexual intercourse, Vail-Smith and Durham ${ }^{11}$ developed the Condom Embarrassment Scale (CES) for use in a group of American university students.

The scale consists of 18 items, with a Likert-type answer format, with five answer options, ranging from totally disagree to totally agree. In these 18 items, three dimensions are evident: the first, which assesses the acquisition, purchase, obtaining or possession of condoms (items 1,2,3,4,5,6, 7 and 12); the second, associated with the negotiation of use with the sexual partnership (items $8,9,10$, 11 and 13); and, the third, related to condom use (items 14,15,16, 17 and 18). The final score varies between 18 and 90 points; scores ranging from 18 to 54 indicate a low level of embarrassment and those between 55 and 90 indicate a higher level of embarrassment in condom use. ${ }^{11-12}$ Currently, CES has already been translated and adapted into the Portuguese language from Portugal by CunhaOliveira et al., which was called the Escala de Embaraço face ao Preservativo ${ }^{12}$.

Considering the absence of instruments to assess embarrassment regarding condom use in Brazil, as well as the absence of Brazilian studies dedicated to analyzing this behavior in university students, this study aimed to carry out the cross-cultural adaptation of CES to the Brazilian context. Research on the topic are relevant because they provide greater knowledge of the reasons for using condoms, as well as supporting policies for the prevention of HIV transmission and other STIs. 


\section{METHOD}

This is a methodological cross-cultural adaptation study ${ }^{13}$ of CES in a sample of Brazilian university students. The procedure consisted of five steps: initial translation, summary of translated versions, reverse translation, expert committee and pre-test, as suggested by the specialized literature. ${ }^{14}$

The cross-cultural adaptation process ensures that the adapted instrument can be used in other cultures, provided that all stages of the process have been completed, and the adjustments of words, expressions and format are made. A flow chart of the entire procedure is presented in Figure 1.

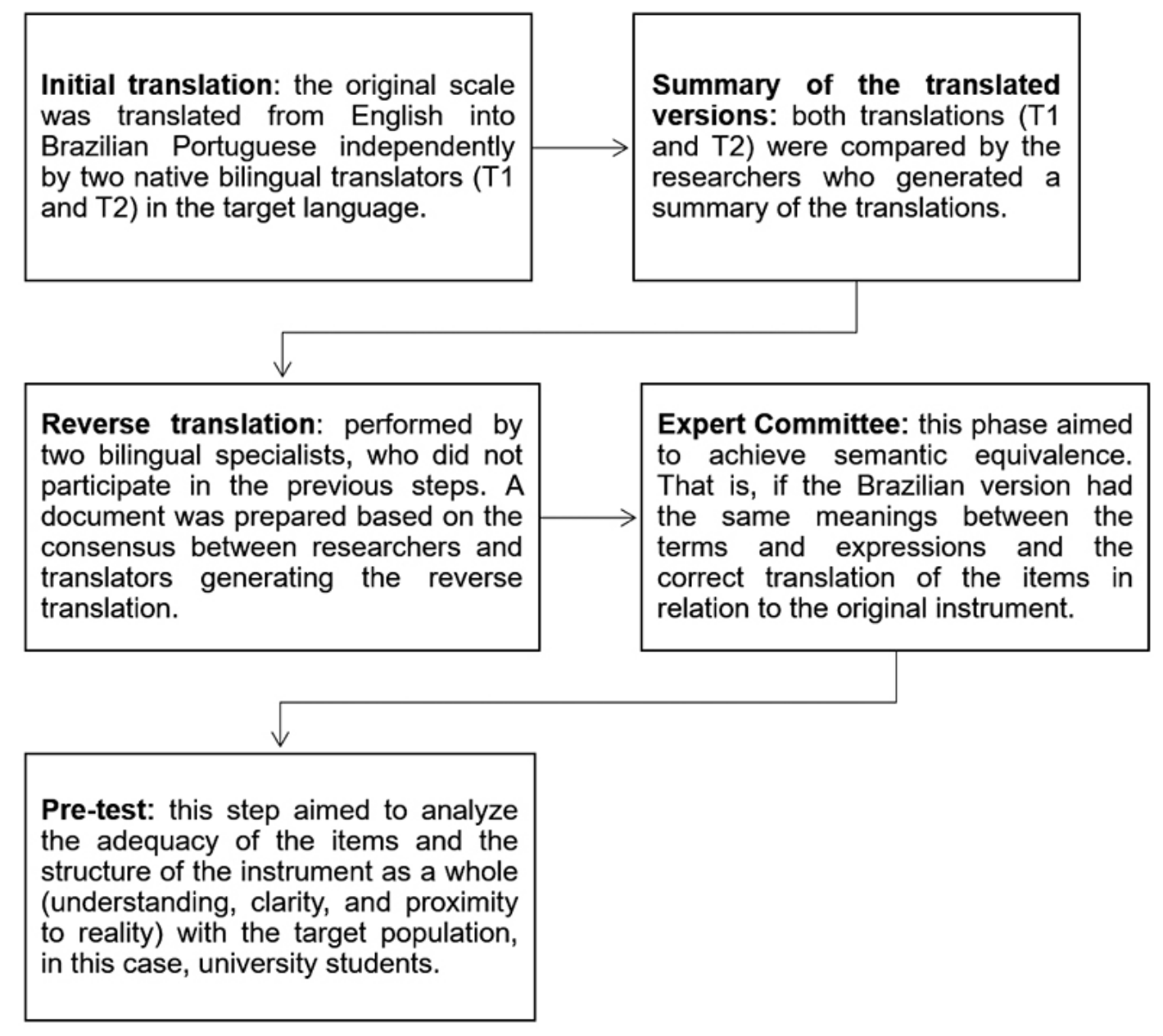

Figure 1 - Flowchart of the Transcultural Adaptation Process. Rio Verde, Goiás, Brazil, 2016.

Initially, contact was made with the original authors of the scale and permission was requested to proceed with the translation and cultural adaptation process for the Brazilian context.

After authorization, the original scale was translated from English into Brazilian Portuguese independently by two native bilingual translators (T1 and T2) in the target language. Translator 1 , was aware of the objectives of the study and was an English language teacher. Translator 2, did not know the objectives of the study, had proficiency in the English language, in addition to developing work on translating scientific articles and materials from English into Brazilian Portuguese. ${ }^{15}$

The translations of T1 and T2 were compared by researchers and translators to obtain a summary of the translations. ${ }^{14}$ In this stage, a careful attempt was made to identify the items translated in different ways in T1 and T2, which were discussed, observing the meaning of words in the English and Brazilian Portuguese languages and the substitution of equivalent terms or expressions and, by means of a consensus (summary), the first translation into Brazilian Portuguese was established. 
Subsequently, this initial translation was submitted to back-translation into English by two bilingual specialists, ${ }^{16}$ who did not participate in the previous steps and who did not know the scale. ${ }^{17}$ These translations were unified through consensus between researchers and translators, resulting in the back-translation.

The version with the back translation was sent to the first author of the original instrument (Dr. Karen Vail-Smith) in order to assess the accuracy of the translated version, ${ }^{18}$ which made it possible to create a new version in Brazilian Portuguese.

This new version was reviewed by a Committee of Experts composed of five professionals, ${ }^{17}$ involving specialists with a command of the English and Brazilian Portuguese; four with professional and academic experience in the area of sexually transmitted infections and one with knowledge in validating instruments. The formation of this committee was aimed at the evaluation of semantic, idiomatic, experiential and conceptual equivalences. ${ }^{18}$

The expert committee evaluated the original instrument and the translations individually and independently, evaluating discrepancies and adequacy of terms.

In order to obtain semantic equivalence, the committee analyzed whether the Brazilian version had the same meanings between the terms and expressions and the correct translation of the items in relation to the original instrument. To analyze the idiomatic equivalence, it was verified whether the colloquial expressions of the source language had equivalent expressions in the target language, without changing the meaning of the item. Experiential equivalence was verified by observing whether the translated items were applicable to Brazilian culture, and conceptual equivalence sought to assess whether the meanings of terms and expressions contained different meanings between English and Brazilian culture. This phase gave rise to the consensual Brazilian version (VBC).

The fifth phase consisted of a pre-test of the consensual Brazilian version in order to analyze the adequacy of the items and the structure of the instrument as a whole (understanding, clarity and proximity to reality) by the target population. This phase was characterized by the previous application of the instrument in a convenience sample composed of 42 undergraduate students from the nursing $(47.62 \%)$ and Dentistry (52.38\%) courses, of both sexes ( $45.24 \%$ male; $54.76 \%$ female), aged between 18 and 28 years, from a university located in the Midwest Region of Brazil. The literature recommends a sample of between 30 and 40 people from the target population. ${ }^{14}$

The participating university students were informed about the objectives of the study. Afterwards, they were asked to respond individually and critically to the 18 VBC questions, being guided by the researchers who asked for clarification in case of doubts and difficulties regarding the lack of terms, problems understanding the items and scale format. They were also asked to verify that the expressions used correspond to those used in the university environment and that they could propose some modification if necessary. Then, after answering the questionnaire, a discussion was held with the group to identify the understanding and clarity of the consensual version, thus obtaining the final Brazilian Portuguese version.

All participants expressed their agreement to participate by signing the Informed Consent Form. The study was approved by the Research Ethics Committee. 


\section{RESULTS}

The stages of translation from English into Brazilian Portuguese and the summary of the translations proved to be similar in relation to the general meaning. The terms and expressions that showed different disagreements or translations were evaluated and modified together with the translators. For the term condom, we opted to use condom and in relation to the term drug store, we opted to use pharmacy, since in Brazil the term pharmacy is less used.

From the summary of the two translations, a summary of the Condom Embarrassment Scale for Brazilian Portuguese was obtained (Chart 1).

Chart 1 - Translations 1 and 2 and the Brazilian Portuguese summary of the Condom Embarrassment Scale (CES). Rio Verde, Goiás, Brazil, 2016.

\begin{tabular}{|c|c|c|c|}
\hline & Translator 1 & Translator 2 & Synthesis \\
\hline Title & $\begin{array}{l}\text { Escala de constrangimento } \\
\text { do preservativo }\end{array}$ & $\begin{array}{c}\text { Escala constrangimento } \\
\text { frente ao uso do preservativo }\end{array}$ & $\begin{array}{l}\text { Escala de constrangimento } \\
\text { no uso do preservativo }\end{array}$ \\
\hline Instruction: & $\begin{array}{l}\text { Instruções: Os itens a seguir } \\
\text { avaliam o quanto você fica } \\
\text { (ou sente) constrangido com } \\
\text { relação ao uso da camisinha. } \\
\text { Usando a seguinte escala, } \\
\text { por favor responda cada um } \\
\text { dos itens abaixo listados. }\end{array}$ & $\begin{array}{l}\text { Instruções: Os itens a seguir } \\
\text { pretendem avaliar como } \\
\text { você se sente ou sentiria } \\
\text { sobre o uso do preservativo. } \\
\text { Responda a cada um dos } \\
\text { itens listados abaixo de } \\
\text { acordo com o item que } \\
\text { melhor representa sua } \\
\text { opinião. }\end{array}$ & $\begin{array}{l}\text { Instruções: Os itens a seguir } \\
\text { pretendem avaliar como } \\
\text { você se sente ou sentiria } \\
\text { sobre o uso do preservativo. } \\
\text { Responda a cada um dos } \\
\text { itens listados abaixo de } \\
\text { acordo com o item que } \\
\text { melhor representa sua } \\
\text { opinião. }\end{array}$ \\
\hline Scale & $\begin{array}{l}A=\text { Discordo com veemência } \\
B=\text { Discordo } \\
C=\underline{\text { Não concordo nem }} \\
\text { discordo } \\
D=\text { Concordo } \\
E=\text { Concordo com veemência }\end{array}$ & $\begin{array}{l}A=\text { Discordo fortemente } \\
B=\text { Discordo } \\
C=\underline{\text { Indeciso }} \\
D=\text { Concordo } \\
E=\text { Concordo fortemente }\end{array}$ & $\begin{array}{l}A=\text { Discordo fortemente } \\
B=\text { Discordo } \\
C=\text { Indeciso } \\
D=\text { Concordo } \\
E=\text { Concordo fortemente }\end{array}$ \\
\hline 01 & $\begin{array}{l}\text { Fico constrangido ou ficaria } \\
\text { constrangido com relação a } \\
\text { comprar preservativo em uma } \\
\text { drogaria perto do campus. }\end{array}$ & $\begin{array}{l}\frac{\text { Fico ou ficaria constrangido }}{\text { se tivesse que comprar uma }} \\
\text { camisinha na drogaria perto } \\
\text { do campus. }\end{array}$ & $\begin{array}{l}\text { Fico constrangido ou ficaria } \\
\text { constrangido se tivesse que } \\
\text { comprar um preservativo na } \\
\text { farmácia perto do campus. }\end{array}$ \\
\hline 02 & $\begin{array}{l}\text { Fico constrangido ou ficaria } \\
\text { constrangido com relação a } \\
\text { comprar preservativo em uma } \\
\text { drogaria perto da casa dos } \\
\text { meus pais. }\end{array}$ & $\begin{array}{l}\text { Fico ou ficaria constrangido } \\
\text { se tivesse que comprar uma } \\
\text { camisinha em uma farmácia } \\
\text { perto de onde meus pais } \\
\text { moram. }\end{array}$ & $\begin{array}{l}\text { Fico constrangido ou ficaria } \\
\text { constrangido se tivesse que } \\
\text { comprar um preservativo em } \\
\text { uma drogaria perto da casa } \\
\text { dos meus pais. }\end{array}$ \\
\hline 03 & $\begin{array}{l}\text { Fico constrangido ou ficaria } \\
\text { constrangido com relação a } \\
\text { comprar preservativo em um } \\
\text { lugar onde eu pudesse ter } \\
\text { certeza de que ninguém que } \\
\text { eu conheça me veria. }\end{array}$ & $\begin{array}{l}\text { Fico constrangido ou ficaria } \\
\text { constrangido com a compra } \\
\text { de uma camisinha onde eu } \\
\text { poderia estar certo de que } \\
\text { ninguém que eu conheça me } \\
\text { veria. }\end{array}$ & $\begin{array}{l}\text { Fico constrangido ou ficaria } \\
\text { constrangido com relação a } \\
\text { comprar preservativo em um } \\
\text { lugar onde eu pudesse ter } \\
\text { certeza de que ninguém que } \\
\text { eu conheça me veria. }\end{array}$ \\
\hline 04 & $\begin{array}{l}\text { Fico constrangido ou ficaria } \\
\text { constrangido com relação } \\
\text { a arranjar preservativo nos } \\
\text { Serviços de Saúde Estudantil } \\
\text { (Enfermaria da Escola). }\end{array}$ & $\begin{array}{l}\text { Fico ou ficaria constrangido } \\
\text { se tivesse que pegar } \\
\text { camisinhas em posto de } \\
\text { saúde dentro da universidade. }\end{array}$ & $\begin{array}{l}\text { Fico constrangido ou ficaria } \\
\text { constrangido em relação } \\
\text { a obter preservativo nos } \\
\text { Serviços de Saúde Estudantil } \\
\text { (Enfermaria da Escola). }\end{array}$ \\
\hline 05 & $\begin{array}{l}\text { Fico constrangido ou ficaria } \\
\text { constrangido em relação } \\
\text { a arranjar preservativo em } \\
\text { posto de saúde. }\end{array}$ & $\begin{array}{l}\text { Fico ou ficaria constrangido } \\
\text { se tivesse que pegar } \\
\text { camisinhas em uma unidade } \\
\text { de saúde. }\end{array}$ & $\begin{array}{l}\text { Fico constrangido ou ficaria } \\
\text { constrangido se tivesse que } \\
\text { pegar preservativos em uma } \\
\text { unidade de saúde. }\end{array}$ \\
\hline
\end{tabular}


Chart 1 - Cont.

\begin{tabular}{|c|c|c|c|}
\hline & Translator 1 & Translator 2 & Synthesis \\
\hline 06 & $\begin{array}{l}\text { Fico constrangido ou ficaria } \\
\text { constrangido com relação } \\
\text { a perguntar onde na loja } \\
\text { ficam os preservativos ao } \\
\text { balconista ou farmacêutico da } \\
\text { drogaria. }\end{array}$ & $\begin{array}{l}\text { Fico ou ficaria constrangido } \\
\text { se tivesse que perguntar } \\
\text { para um farmacêutico ou } \\
\text { atendente da farmácia onde } \\
\text { ficam as camisinhas. }\end{array}$ & $\begin{array}{l}\text { Fico constrangido ou ficaria } \\
\text { constrangido se tivesse } \\
\text { que perguntar para um } \\
\text { farmacêutico ou atendente } \\
\text { da farmácia onde ficam os } \\
\text { preservativos. }\end{array}$ \\
\hline 07 & $\begin{array}{l}\text { Fico constrangido ou ficaria } \\
\text { constrangido com relação a } \\
\text { fazer perguntas relacionadas } \\
\text { ao uso do preservativo ao } \\
\text { médico ou profissional da } \\
\text { saúde. }\end{array}$ & $\begin{array}{l}\text { Fico ou ficaria constrangido } \\
\text { em conversar assuntos sobre } \\
\text { o uso da camisinha a um } \\
\text { médico ou outro profissional } \\
\text { da saúde }\end{array}$ & $\begin{array}{l}\text { Fico constrangido ou ficaria } \\
\text { constrangido em fazer } \\
\text { perguntas sobre o uso do } \\
\text { preservativo a um médico ou } \\
\text { outro profissional da saúde. }\end{array}$ \\
\hline 08 & $\begin{array}{l}\text { Fico constrangido ou ficaria } \\
\text { constrangido com relação a } \\
\text { interromper as preliminares } \\
\text { e pedir ao meu parceiro que } \\
\text { coloque o preservativo. }\end{array}$ & $\begin{array}{l}\text { Fico ou ficaria constrangido } \\
\text { se tivesse que durante as } \\
\text { preliminares sexuais pedir ao } \\
\text { meu parceiro (a) para usar a } \\
\text { camisinha. }\end{array}$ & $\begin{array}{l}\text { Fico constrangido ou ficaria } \\
\text { constrangido se tivesse } \\
\text { que durante as preliminares } \\
\text { sexuais pedir ao meu parceiro } \\
\text { para usar o preservativo. }\end{array}$ \\
\hline 09 & $\begin{array}{l}\text { Eu ficaria constrangido se um } \\
\text { novo parceiro insistisse que } \\
\text { nós usássemos preservativo. }\end{array}$ & $\begin{array}{l}\text { Fico ou ficaria constrangido } \\
\text { se um novo parceiro (a) } \\
\text { insistisse para usarmos } \\
\text { camisinha. }\end{array}$ & $\begin{array}{l}\text { Eu ficaria constrangido se um } \\
\text { novo parceiro insistisse que } \\
\text { nós usássemos preservativo. }\end{array}$ \\
\hline 10 & $\begin{array}{l}\text { Fico constrangido ou ficaria } \\
\text { constrangido em dizer ao } \\
\text { meu parceiro, durante as } \\
\text { preliminares, que não estou } \\
\text { disposto a ter relações } \\
\underline{\text { sexuais a não ser que }} \\
\text { usemos preservativo. }\end{array}$ & $\begin{array}{l}\text { Fico ou ficaria constrangido } \\
\text { se durante as preliminares } \\
\text { sexuais tivesse que falar } \\
\text { ao meu parceiro que não } \\
\text { teria relação sexual se não } \\
\text { usarmos uma camisinha. }\end{array}$ & $\begin{array}{l}\text { Fico constrangido ou ficaria } \\
\text { constrangido em dizer ao } \\
\text { meu parceiro, durante as } \\
\text { preliminares sexuais, que não } \\
\text { estou disposto a ter relações } \\
\text { sexuais a não ser que } \\
\text { usemos preservativo. }\end{array}$ \\
\hline 11 & $\begin{array}{l}\text { Fico constrangido ou ficaria } \\
\text { constrangido com relação a } \\
\text { estar preparado e fornecer } \\
\text { o preservativo quando fizer } \\
\text { amor e meu parceiro não tiver } \\
\text { uma. }\end{array}$ & $\begin{array}{l}\begin{array}{l}\text { Fico ou ficaria constrangido } \\
\text { de ter uma camisinha e pedir }\end{array} \\
\text { para usá-lo caso o meu } \\
\text { parceiro não tivesse. }\end{array}$ & $\begin{array}{l}\text { Fico constrangido ou ficaria } \\
\text { constrangido por estar } \\
\text { preparado e fornecer um } \\
\text { preservativo caso o meu } \\
\text { parceiro não tivesse um. }\end{array}$ \\
\hline 12 & $\begin{array}{l}\text { Fico constrangido ou ficaria } \\
\text { constrangido com relação a } \\
\text { carregar um preservativo na } \\
\text { carteira/bolsa. }\end{array}$ & $\begin{array}{l}\text { Fico ou ficaria constrangido } \\
\text { em carregar uma camisinha } \\
\text { em minha carteira ou bolsa. }\end{array}$ & $\begin{array}{l}\text { Fico constrangido ou ficaria } \\
\text { constrangido em carregar } \\
\text { um preservativo em minha } \\
\text { carteira ou bolsa. }\end{array}$ \\
\hline 13 & $\begin{array}{l}\text { Fico constrangido ou ficaria } \\
\text { constrangido em relação a } \\
\text { conversar com meu parceiro } \\
\text { sobre o que penso e sinto } \\
\text { relacionado ao preservativo. }\end{array}$ & $\begin{array}{l}\text { Fico ou ficaria constrangido } \\
\text { em falar com o meu parceiro } \\
\text { sobre o que penso e sinto } \\
\text { sobre o uso da camisinha. }\end{array}$ & $\begin{array}{l}\text { Fico constrangido ou ficaria } \\
\text { constrangido em falar com } \\
\text { o meu parceiro sobre o que } \\
\text { penso e sinto sobre o uso do } \\
\text { preservativo. }\end{array}$ \\
\hline 14 & $\begin{array}{l}\text { Fico constrangido ou ficaria } \\
\text { constrangido se meu parceiro } \\
\text { me assistisse descartar um } \\
\text { preservativo depois de o } \\
\text { termos usado. }\end{array}$ & $\begin{array}{l}\text { Fico ou ficaria constrangido } \\
\text { se o meu parceiro me visse } \\
\text { com uma camisinha na mão } \\
\text { depois que termos usado. }\end{array}$ & $\begin{array}{l}\text { Fico constrangido ou ficaria } \\
\text { constrangido se meu parceiro } \\
\text { me visse descartar um } \\
\text { preservativo depois de o } \\
\text { termos usado. }\end{array}$ \\
\hline 15 & $\begin{array}{l}\text { Fico constrangido ou ficaria } \\
\text { constrangido com relação } \\
\text { a assistir meu parceiro } \\
\text { colocar um preservativo oU } \\
\text { se meu (minha) parceiro (a) } \\
\text { me assistisse colocar um } \\
\text { preservativo. }\end{array}$ & $\begin{array}{l}\text { Fico ou ficaria constrangido } \\
\text { se o meu parceiro me visse } \\
\underline{\text { colocar uma camisinha. }}\end{array}$ & $\begin{array}{l}\text { Fico constrangido ou } \\
\text { ficaria constrangido em } \\
\text { ver meu parceiro colocar } \\
\text { o preservativo OU se meu } \\
\text { parceiro me visse colocar o } \\
\text { preservativo. }\end{array}$ \\
\hline
\end{tabular}


Chart 1 - Cont.

\begin{tabular}{|c|c|c|c|}
\hline & Translator 1 & Translator 2 & Synthesis \\
\hline 16 & $\begin{array}{l}\text { Fico constrangido ou ficaria } \\
\text { constrangido em ajudar } \\
\text { meu parceiro a colocar um } \\
\text { preservativo OU se minha } \\
\text { parceira me ajudasse a } \\
\text { colocar um preservativo. }\end{array}$ & $\begin{array}{l}\text { Fico ou ficaria constrangido } \\
\text { em ajudar o meu parceiro } \\
\text { colocar uma camisinha em } \\
\underline{\text { mim ou se tivesse que ajudá- }} \\
\underline{\text { lo a colocar. }}\end{array}$ & $\begin{array}{l}\text { Fico constrangido ou ficaria } \\
\text { constrangido em ajudar } \\
\text { meu parceiro a colocar um } \\
\text { preservativo OU se meu } \\
\text { parceiro me ajudasse a } \\
\text { colocar um preservativo. }\end{array}$ \\
\hline 17 & $\begin{array}{l}\text { Fico constrangido ou ficaria } \\
\text { constrangido com relação a } \\
\text { assistir meu parceiro remover } \\
\text { um preservativo OU se } \\
\text { minha parceira me assistisse } \\
\text { remover um preservativo. }\end{array}$ & $\begin{array}{l}\text { Fico constrangido ou ficaria } \\
\text { constrangido se o meu } \\
\text { parceiro me visse retirando } \\
\text { uma camisinha. }\end{array}$ & $\begin{array}{l}\text { Fico constrangido ou } \\
\text { ficaria constrangido ao ver } \\
\text { meu parceiro retirar um } \\
\text { preservativo OU se meu } \\
\text { parceiro me visse retirar um } \\
\text { preservativo. }\end{array}$ \\
\hline 18 & $\begin{array}{l}\text { Fico constrangido ou ficaria } \\
\text { constrangido em ajudar } \\
\text { meu parceiro retirar um } \\
\text { preservativo OU se minha } \\
\text { parceira me ajudasse a retirar } \\
\text { um preservativo. }\end{array}$ & $\begin{array}{l}\text { Fico ou ficaria constrangido } \\
\text { em ajudar o meu parceiro a } \\
\text { retirar a camisinha }\end{array}$ & $\begin{array}{l}\text { Fico constrangido ou ficaria } \\
\text { constrangido em ajudar } \\
\text { meu parceiro retirar um } \\
\text { preservativo OU se meu } \\
\text { parceiro me ajudasse a retirar } \\
\text { um preservativo. }\end{array}$ \\
\hline
\end{tabular}

Note: The underline represents the discrepancies between translations 1 and 2 and the final summary.

After the summary and adjustments, the Brazilian version was translated back into English (back-translation), obtaining a new English version. This version was sent to the first author of the original scale for evaluation. The author made suggestions for adjustments in items 1,5,6,8, 11 and 15 , which considered that the translation had differed from the original idea (Chart 2).

Chart 2 - Comparison of the original scale and version with back translation. Rio Verde, Goiás, Brazil, 2016.

\begin{tabular}{|c|l|l|}
\hline Title & \multicolumn{1}{|c|}{ Original scale } & \multicolumn{1}{|c|}{ Back translation version } \\
\hline & Condom Embarrassment Scale & Scale of Embarrassment on condom use \\
\hline \multirow{5}{*}{ Instructors } & $\begin{array}{l}\text { The following items assess how embarrassed } \\
\text { you do feel (or would feel) about condom use. } \\
\text { Using the following scale, please respond to } \\
\text { each of the items listed below. }\end{array}$ & $\begin{array}{l}\text { The following items intend to evaluate how } \\
\text { you feel (or would feel) regarding the use of } \\
\text { condoms. Read each statement carefully and } \\
\text { mark with an x the option that best reflects } \\
\text { your opinion, according to the scale: }\end{array}$ \\
\hline \multirow{5}{*}{ Scale } & $\begin{array}{l}\text { A = Strongly disagree } \\
\text { B = Disagree } \\
\text { C = Neither agree or disagree } \\
\text { D = Agree } \\
\text { E = Strongly agree }\end{array}$ & $\begin{array}{l}\text { A = Strongly disagree; } \\
\text { B = Disagree; } \\
\text { C = Neither agree nor disagree; } \\
\text { D = Agree; } \\
\text { E = Strongly agree. }\end{array}$ \\
\hline \multirow{2}{*}{01} & $\begin{array}{l}\text { I am embarrassed or would be embarrassed } \\
\text { about buying a condom from a drug store near } \\
\text { campus. }\end{array}$ & $\begin{array}{l}\text { I get embarrassed or would get embarrassed } \\
\text { If I had to buy condoms in pharmacies or } \\
\text { supermarkets near the university. }\end{array}$ \\
\hline \multirow{2}{*}{03} & $\begin{array}{l}\text { I am embarrassed or would be embarrassed } \\
\text { about buying a condom from a drug store } \\
\text { close to where my parents live. }\end{array}$ & $\begin{array}{l}\text { I get embarrassed or would get embarrassed } \\
\text { if I had to buy condoms in pharmacies or } \\
\text { supermarkets near my parents' house. }\end{array}$ \\
\cline { 2 - 4 } & $\begin{array}{l}\text { I am embarrassed or would be embarrassed } \\
\text { about buying a condom from a place where I } \\
\text { could be certain no onel know would see me. }\end{array}$ & $\begin{array}{l}\text { I get embarrassed or would get embarrassed } \\
\text { about buying condoms in a place where I } \\
\text { could assure nobody I know would see me. }\end{array}$ \\
\hline \multirow{2}{*}{04} & $\begin{array}{l}\text { I am embarrassed or would be embarrassed } \\
\text { about obtaining condoms from Student Health } \\
\text { Services (School Infirmary). }\end{array}$ & $\begin{array}{l}\text { I get or would get embarrassed about getting a } \\
\text { condom in a school and / or university's ward. }\end{array}$ \\
\hline
\end{tabular}


Chart 2 - Cont.

\begin{tabular}{|c|c|c|}
\hline Title & Original scale & Back translation version \\
\hline 05 & $\begin{array}{l}\text { I am embarrassed or would be embarrassed } \\
\text { about obtaining condoms from a local health } \\
\text { department. }\end{array}$ & $\begin{array}{l}\text { I get embarrassed or would get embarrassed if } \\
\text { I had to get condoms in a health center. }\end{array}$ \\
\hline 06 & $\begin{array}{l}\text { I am embarrassed or would be embarrassed } \\
\text { about asking a pharmacist or drug store clerk } \\
\text { where condoms are located in the store. }\end{array}$ & $\begin{array}{l}\text { I get embarrassed or would get embarrassed } \\
\text { if I had to ask a pharmacist or a pharmacy } \\
\text { attendant where the condoms are in the store. }\end{array}$ \\
\hline 07 & $\begin{array}{l}\text { I am embarrassed or would be embarrassed } \\
\text { about asking a doctor or other health care } \\
\text { professional questions about condom use. }\end{array}$ & $\begin{array}{l}\text { I get embarrassed or would get embarrassed } \\
\text { to talk about the use of condoms with a doctor } \\
\text { or other health care provider. }\end{array}$ \\
\hline 08 & $\begin{array}{l}\text { I am embarrassed or would be embarrassed } \\
\text { about stopping during foreplay and asking my } \\
\text { partner to use a condom. }\end{array}$ & $\begin{array}{l}\text { I get embarrassed or would get embarrassed if } \\
\text { I had to interrupt foreplay to ask my partner to } \\
\text { use a condom. }\end{array}$ \\
\hline 09 & $\begin{array}{l}\text { I would be embarrassed if a new partner } \\
\text { insisted that we use a condom. }\end{array}$ & $\begin{array}{l}\text { I would get embarrassed if a new partner } \\
\text { insisted that we used a condom. }\end{array}$ \\
\hline 10 & $\begin{array}{l}\text { I am embarrassed or would be embarrassed } \\
\text { to tell my partner during foreplay that I am not } \\
\text { willing to have sexual intercourse unless we } \\
\text { use a condom. }\end{array}$ & $\begin{array}{l}\text { I get embarrassed or would get embarrassed } \\
\text { to tell my partner, during foreplay, that I am not } \\
\text { willing to have sexual intercourse unless we } \\
\text { use a condom. }\end{array}$ \\
\hline 11 & $\begin{array}{l}\text { I am embarrassed or would be embarrassed } \\
\text { about being prepared and providing a condom } \\
\text { during lovemaking if my partner didn't have } \\
\text { one. }\end{array}$ & $\begin{array}{l}\text { I get embarrassed or would get embarrassed } \\
\text { about being prepared and provide a condom } \\
\text { in case my partner did not have one. }\end{array}$ \\
\hline 12 & $\begin{array}{l}\text { I am embarrassed or would be embarrassed } \\
\text { about carrying a condom around in my wallet } \\
\text { / purse. }\end{array}$ & $\begin{array}{l}\text { I get embarrassed or would get embarrassed } \\
\text { to carry a condom in my wallet or purse. }\end{array}$ \\
\hline 13 & $\begin{array}{l}\text { I am embarrassed or would be embarrassed } \\
\text { about talking to my partner about my thoughts } \\
\text { and feelings about condom use. }\end{array}$ & $\begin{array}{l}\text { I get embarrassed or would get embarrassed } \\
\text { to talk with my partner about what I think and } \\
\text { feel regarding condom use. }\end{array}$ \\
\hline 14 & $\begin{array}{l}\text { I am embarrassed or would be embarrassed if } \\
\text { my partner watched me dispose of a condom } \\
\text { after we had used it. }\end{array}$ & $\begin{array}{l}\text { I get embarrassed or would get embarrassed if } \\
\text { my partner saw me discard a condom after we } \\
\text { used it. }\end{array}$ \\
\hline 15 & $\begin{array}{l}\text { I am embarrassed or would be embarrassed } \\
\text { about watching my partner put on a condom } \\
\text { OR if my partner watched put me on a } \\
\text { condom. }\end{array}$ & $\begin{array}{l}\text { I get or would get embarrassed if my partner } \\
\text { observed me putting a condom or if my partner } \\
\text { saw me put a condom. }\end{array}$ \\
\hline 16 & $\begin{array}{l}\text { I am embarrassed or would be embarrassed } \\
\text { about helping my partner put on a condom OR } \\
\text { if my partner helped me put on a condom. }\end{array}$ & $\begin{array}{l}\text { I get embarrassed or would get embarrassed } \\
\text { to help my partner put a condom OR if my } \\
\text { partner helped me put a condom. }\end{array}$ \\
\hline 17 & $\begin{array}{l}\text { I am embarrassed or would be embarrassed } \\
\text { about watching my partner remove a condom } \\
\text { OR if my partner watched remove me a } \\
\text { condom. }\end{array}$ & $\begin{array}{l}\text { I get embarrassed or would get embarrassed } \\
\text { to see my partner remove a condom OR if my } \\
\text { partner saw removes me a condom. }\end{array}$ \\
\hline 18 & $\begin{array}{l}\text { I am embarrassed or would be embarrassed } \\
\text { about helping my partner remove a condom } \\
\text { OR if my partner helped me remove a } \\
\text { condom. }\end{array}$ & $\begin{array}{l}\text { I get embarrassed or would get embarrassed } \\
\text { to help my partner remove a condom OR if my } \\
\text { partner helped me remove a condom. }\end{array}$ \\
\hline
\end{tabular}

Note: the underline represents points that differed from the original idea of the instrument. 
Regarding items 1,2,5, 6 and 8, the author highlighted the need to emphasize that the scale assesses the embarrassment when obtaining the condom, as a routine behavior. As it was written: "if I had to buy a condom", for the author, it implied that the young man did not want to buy or did not have the habit, but was compelled to do so. In item 11, the author requested the inclusion of the expression "during sex". The translated item omitted that the occasion was during sex.

All of the author's suggestions were included in the scale, generating the second Brazilian version, which was submitted for analysis by the expert committee.

During the review of the committee, semantic, idiomatic and experiential equivalences were evaluated. Conceptual equivalence was obtained both by the committee's evaluation and by the pretest. This stage gave rise to the consensual Brazilian version.

The changes made by the committee started with the title considering that the term "condom" is suitable for translation, but in Brazil the term condom is used. We chose to use the expression "condom (condom)" in the title and the entire scale and wait for the evaluation by the target audience to choose the term that would provide greater clarity to young people.

Letters were replaced by numbers, in the form of the Likert scale response, $A=$ Strongly disagree; $\mathrm{B}=$ Disagree $; \mathrm{C}=$ Neither agree or disagree; $\mathrm{D}=$ Agree; $\mathrm{E}=$ Strongly agree, understanding that the use of numbers can facilitate the completion of the scale and has more similarity with intensity, in addition to the use of the word "totally": 1 = Totally disagree; $2=$ Disagree; $3=$ I neither agree nor disagree; 4 = I agree; 5 = I totally agree. The word fully is used in several Brazilian instruments and is easy to understand.

In item 1, to facilitate understanding and because it is more familiar, the word campus was changed to "university" and the term "supermarket" was included, since in Brazil condoms are sold in pharmacies and supermarkets.

In item 3, the expression "buying a condom from a place where I could be sure that nobody I know would see me", to facilitate the understanding of the statement was modified to "buy condoms in a place where I could be sure that no one I know would see me".

In item 4, the term school infirmary, was changed to "school and/or university infirmary" for better understanding by the target population. Although in Brazilian universities there are rarely any infirmaries, the committee opted to maintain the statement.

In item 7 , in relation to the term questions about condom use, in the translated version, "asking questions related to condom use" was used, but to designate a dialogue with the doctor or another health professional, it was modified to "talk about condom use with a doctor or other health professional ".

In item 10, there was a consensus that there would be no need for the expression "sexual foreplay" to characterize the moment that precedes the sexual act and avoid the repetition of the sexual word, choosing to keep only "during the foreplay, which I am not willing to having sex".

In the pre-test, all 42 university students $(100 \%)$ responded to the scale, classifying it as containing very clear instructions. Most participants, 32 (78\%), classified the questions as easy to understand and $10(22 \%)$ as regular comprehension. However, everyone considered the scale to be very repetitive. As a suggestion, it was requested to use the term "camisinha" instead of the term "condom" throughout the scale, as it is a term that is more widely disseminated among the target audience, in this case, university students. The university students were also questioned if there was a need to use the term partner on the scale to facilitate understanding, which everyone considered unnecessary, and should only use the term "partner" to designate a man or woman.

After the adjustments were made, the final Brazilian Portuguese version was obtained, which was called the Condom Embarrassment Scale - CEP (Chart 3). 
Chart 3 - Brazilian version of the Condom Embarrassment Scale. Rio Verde, Goiás, Brazil, 2016.

Os itens a seguir pretendem avaliar como você se sente ou sentiria sobre o uso da camisinha. Leia com atenção cada uma das afirmativas e assinale com um $X$ a opção que melhor traduz sua opinião, de acordo com a seguinte escala:

1-Discordo totalmente; 2-Discordo; 3-Não concordo, nem discordo; 4-Concordo; 5-Concordo totalmente.

\begin{tabular}{|l|l|l|l|l|l|}
\hline & $\mathbf{1}$ & $\mathbf{2}$ & $\mathbf{3}$ & $\mathbf{4}$ & $\mathbf{5}$ \\
\hline $\begin{array}{l}\text { 1. Fico constrangido ou ficaria constrangido ao comprar camisinha na } \\
\text { farmácia ou supermercado perto da universidade. }\end{array}$ & & & & & \\
\hline 2. Fon & & &
\end{tabular}

2. Fico constrangido ou ficaria constrangido ao comprar uma camisinha em uma farmácia ou supermercado perto da casa dos meus pais.

3. Fico constrangido ou ficaria constrangido ao comprar camisinha em um lugar onde eu pudesse ter certeza de que ninguém que eu conheça me veria.

4. Fico constrangido ou ficaria constrangido em pegar camisinha em uma enfermaria da universidade.

5. Fico constrangido ou ficaria constrangido ao pegar camisinhas em uma unidade de saúde.

6. Fico constrangido ou ficaria constrangido ao perguntar para um farmacêutico ou atendente da farmácia onde ficam as camisinhas.

7. Fico constrangido ou ficaria constrangido em conversar sobre o uso da camisinha com um médico ou outro profissional da saúde.

8. Fico constrangido ou ficaria constrangido ao interromper as preliminares sexuais para pedir ao meu parceiro que use camisinha.

9. Eu ficaria constrangido se um novo parceiro insistisse que nós usássemos camisinha.

10. Fico constrangido ou ficaria constrangido em dizer ao meu parceiro, durante as preliminares que não estou disposto a ter relações sexuais a não ser que usemos camisinha.

11. Fico constrangido ou ficaria constrangido em estar preparado durante o sexo e ter uma camisinha caso o meu parceiro não tivesse uma.

12. Fico constrangido ou ficaria constrangido em carregar uma camisinha em minha carteira ou bolsa.

13. Fico constrangido ou ficaria constrangido em falar com o meu parceiro sobre o que penso e sinto sobre o uso da camisinha.

14. Fico constrangido ou ficaria constrangido se meu parceiro me visse descartar uma camisinha depois de a termos usado.

15. Fico constrangido ou ficaria constrangido em ver meu parceiro colocar a camisinha ou se meu parceiro me visse colocar a camisinha.

16. Fico constrangido ou ficaria constrangido em ajudar meu parceiro_a colocar uma camisinha OU se meu parceiro me ajudasse a colocar uma camisinha.

17. Fico constrangido ou ficaria constrangido ao ver meu parceiro_retirar uma camisinha OU se meu parceiro me visse retirar uma camisinha.

18. Fico constrangido ou ficaria constrangido em ajudar meu parceiro. retirar uma camisinha OU se meu parceiro me ajudasse a retirar uma camisinha. 


\section{DISCUSSION}

The CES translation and cultural adaptation process was carried out according to the methodology suggested in the literature. ${ }^{14,17-18}$ This process is characterized by a careful methodology, with the aim of achieving the maximum correspondence between the original instrument and the version translated, prioritizing the search for a cultural approach and an adaptation of language to the population to whom the instrument is addressed. ${ }^{13,19}$

In the initial and back translation stages, two independent translations were performed for the detection of errors and divergent interpretations of ambiguous items in the original instrument, in order to eliminate linguistic and cultural bias. ${ }^{1}$ The literature recommends that the translation is performed by at least two independent translators, qualified professionals and that their mother tongue is the target language. ${ }^{20}$

The translating process from one language to another must consider the language, the cultural context and the lifestyle, observing the technical, linguistic and semantic aspects. ${ }^{21}$ Changes in the format of the original instrument should not occur in the translation and back-translation stages, as addition or exclusion of items, as it may compromise its applicability and equivalence with the original instrument, ${ }^{22}$ since the translation of an instrument alone does not guarantee its applicability. ${ }^{21}$

In the back-translation stage, the translators must not have participated in the previous stage, they must be bilingual and not know the objectives of the study, reducing the possibility of generating prejudices and expectations. ${ }^{23}$

The back translation, when sent to the author of the original scale evaluated the accuracy of the translated version and an additional quality control check of all semantic and idiomatic adjustment procedures. Thus, it can be said that the CEP in Portuguese maintained the coherence and integration of the items.

It is important to mention that the meanings attributed to facilitate the understanding of the scale by the target population in the evaluation of the committee of experts, gave the university students a good understanding and acceptance of the items, thus ensuring unanimity and correspondence with the Brazilian cultural context, preserving the contents of the original scale. In other words, the participation of specialists was essential for a thorough evaluation, without any change in the essence of the instrument. This procedure corroborates the literature on cross-cultural adaptation of psychometric instruments. ${ }^{24}$

The committee's evaluation took into account the knowledge of the target culture, in which the approximation of the Brazilian reality was sought, such as the inclusion of "supermarkets" in the scale.

In the responses to the items on the scale, the Likert-type format was maintained with the same number of response options with only one change, from letters to numbers, to express a greater intensity relationship. The committee can modify the initial guidelines and format of the instrument, change or reject inappropriate items so that the instrument is truly understandable to the target audience. ${ }^{25}$

The evaluation by the target audience in the pre-test stage allowed greater methodological rigor in the cross-cultural adaptation process of the instrument. This procedure allowed the analysis of the adequacy of the items and the structure of the instrument, in addition to verifying whether the terms are clear and in accordance with reality, as well as verifying practical aspects of their application. ${ }^{13-14,21}$

The evaluation performed by the target audience, according to Guillemin (1995), ${ }^{23}$ can be performed by applying the instrument to a small group of the target population, with the aim of identifying words or expressions that are difficult to understand, assessing acceptability and requesting comments on the instrument in general.

Thus, the target audience consisted of university students in a very wide age group and for which the instrument is intended, from 18 to 28 years old, so that the translated and adapted version 
can be applied in different populations of university students and in different locations. There was a good acceptability of the scale, and there was no doubt about the items or meanings.

The comprehension and clarity reported by the target audience during the pre-test stage demonstrated that the stages of the cross-cultural adaptation process were successfully completed, which resulted in the final version of the instrument.

Additional studies aimed at investigating the psychometric properties and other forms of validity of the scale are necessary for its application in the Brazilian context.

\section{CONCLUSION}

The CES's cross-cultural adaptation process aimed to achieve the maximum correspondence between the original and the translated scale, prioritizing idiomatic, cultural and methodological aspects. The steps taken during the process allowed the necessary adaptations to be made to the socio-cultural reality and the level of understanding of the target population. Thus, the Brazilian Portuguese version, called the Embarrassment Scale for the use of Condoms, proved to be well understood when applied to university students. The results of the translation and cross-cultural adaptation demonstrated that the CES maintained equivalence with the North American version.

\section{REFERENCES}

1. Smith L, Angarone MP. Sexually Transmitted Infections. Urol Clin N Am [Internet]. 2015 [cited 2019 June 26];42(4):507-18. Available from: https://doi.org/10.1016/j.ucl.2015.06.004

2. World Health Organization. WHO Sexually Transmitted Infections (STIs): key facts [Internet]. Geneva(CH): WHO; 2016. Available from: http://www.who.int/mediacentre/factsheets/fs110/en/

3. Newman L, Rowley J, Hoorn S Vander, Wijesooriya NS, Unemo M, Low N, et al. Global estimatives of the prevalence and incidence of four curable sexually transmitted infections in 2012 based on systematic review and global reporting. Plos One [Internet]. 2015 [cited 2019 Jan 16];10(12):e0143304. Available from: https://doi.org/10.1371/journal.pone.0143304

4. World Health Organization. Sexual and reproductive health Global health sector strategy on Sexually Transmitted Infections. 2016-2021. Geneva (CH): WHO; 2016. Available from: https:// apps.who.int/iris/bitstream/handle/10665/246178/WHO-HIV-2016.05-eng.pdf

5. Brasil. Ministério da Saúde. Departamento de Vigilância, Prevenção e Controle das Infecções Sexualmente Transmissíveis, do HIV/Aids e das Hepatites Virais. Boletim Epidemiológico 20172018. Brasília, DF (BR): Ministério da Saúde, 2018. Available from: http://www.aids.gov.br/pt-br/ pub/2018/boletim-epidemiologico-hivaids-2018

6. Carneiro R, Silva N, Alves T, Albuquerque D, Brito D, Oliveira L. Educação sexual na adolescência: uma abordagem no contexto escolar. Sanare [Internet]. 2015 [cited 2018 Dec 12];14(1):104-8. Available from: https://snare.emnuves.com.br/sanare/article/view/617/334

7. Fonte VRF, Spindola T, Francisco MTR, Sodré CP, André NLNO, Pinheiro CDP. Young university students and the knowledge about sexually transmitted infections. Esc Anna Nery [Internet]. 2018 [cited 2017 Mar 04];22(2):e20170318. Available from: https://doi.org/10.1590/2177-9465ean-2017-0318

8. Bertoli R, Scheidmantel C, De-Carvalho N. College students and HIV infection : a study of sexual behavior and vulnerabilities 1. Brazilian J Sex Transm Dis [Internet]. 2016 [cited 2019 Jul 04]; 28(3):90-5. Available from: http://www.dst.uff.br/revista28-3-2016/DST v28n3_IN_90-95.pdf

9. Moreira LR, Dumith SC, Paludo SS. Condom use in last sexual intercourse among undergraduate students: how many are using them and who are they? Ciênc Saúde Coletiva [Internet]. 2018 [cited 2019 Jul 04];23(4):1255-66. Available from: https://doi.org/10.1590/1413-81232018234.16492016 
10. Campbell C, Lin H, Fulp W, Papenfuss M, Salmerón J, Quiterio M, et al. Consistent condom use reduces the genital human papillomavirus burden among high-risk men: The HPV infection in men study. J Infect Dis [Internet]. 2013 [cited 2018 Nov 11];208(3):373-84. Available from: https://doi.org/10.1093/infdis/jit191

11. Vail-Smith, K.; Durham T. A scale to measure embarrassment associated with condom use. J Heal Educ [Internet]. 1992 [cited 2015 Mai 12]; 23(4):209-14. Available from: https://doi.org/10. 1080/10556699.1992.10616293

12. Cunha-Oliveira A, Cardoso IM, Cardoso SM. Adaptação para a Língua Portuguesa da Escala de Embaraço face ao Preservativo. Interações [Internet]. 2011 [cited 2015 May 12];11(20):133-50. Available from: https://doi.org/10.1080/10556699.1992.10616293

13. Arafat S, Chowdhury H, Qusar M, Hafez M. Cross Cultural Adaptation and Psychometric Validation of Research Instruments: a Methodological Review. J Behav Heal [Internet]. 2016 [cited 2015 May 11]:5(3):129. Available from: https://doi.org/110.5455/jbh.20160615121755

14. Beaton D, Bombardier C, Guillemin F, Ferraz M. Guidelines for the process of Cross Cultural adaptation of Self Report mesures. Spine [Internet]. 2000 [cited 2015 Mai 11];25(24):3186-91.

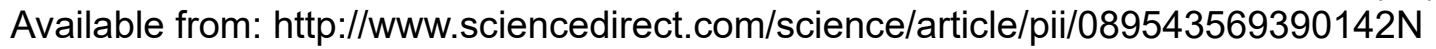

15. Guillemin F, Bombardier C, Beaton D. Cross-cultural adaptation of health-related quality of life measures: Literature review and proposed guidelines. J Clin Epidemiol [Internet]. 1993 [cited 2015 Mai 11];46(12):1417-32. Available from: http://www.sciencedirect.com/science/article/ pii/089543569390142N

16. Oliveira F, Kuznier TP, Souza CC, Chianca TCM. Theoretical and methodological aspects for the cultural adaptation and validation of instruments in nursing. Texto Contexto Enferm [Internet]. 2018 [cited 2019 Jul 04];27(2):e4900016. Available from: https://doi.org/10.1590/0104070720180004900016

17. Tuthill EL, Butler LM, McGrath JM, Cusson RM, Makiwane GN, Gable RK, et al. Cross-cultural adaptation of instruments assessing breastfeeding determinants: A multi-step approach. Int Breastfeed J [Internet]. 2014 [cited 2015 May 28];9:16. Available from: https://doi.org/10.1186/17464358-9-16

18. Borsa J, Damásio B, Bandeira D. Adaptação e Validação de Instrumentos Psicológicos entre Culturas : Algumas Considerações. Paidéia [Internet]. 2012 [cited 2015 May 27];22(53):423-32. Available from: https://doi.org/10.1590/S0103-863X2012000300014

19. Bortoli P, Jacob E, Castral T, Santos C, Fernandes A, Nascimento L. Cross-cultural adaptation of the adolescent pediatric pain tool for brazilian children and adolescents with cancer. Texto Contexto Enferm [Internet]. 2019 [cited 2019 Jul 03];28:e20160108. Available from: https://doi. org/10.1590/1980-265X-TCE-2016-0108

20. Alexandre NMC, Guirardello EDB. Adaptación cultural de instrumentos utilizados en salud ocupacional. Rev Panam Salud Publica. [Internet]. 2002 [cited 2016 Mar 13];11(2):109-11. Available from: https://www.scielosp.org/pdf/rpsp/2002.v11n2/109-111

21. Cassep-Borges V, Balbinotti M, Teodoro M. Tradução e Validação de conteúdo: uma proposta para a adaptação de intrumentos. In: Pasquali, L. Instrumentação psicológica: Fundamentos e práticas. Porto Alegre, RS (BR): Artmed; 2010. p. 506-20.

22. Alexandre NMC, Coluci MZO. Validade de conteúdo nos processos de construção e adaptação de instrumentos de medidas. Cien Saude Colet [Internet]. 2011 [cited 2016 Jan 31];16(7):3061-8. Available from: http://www.scielo.br/scielo.php?script=sci_arttext\&pid=S1413$81232011000800006 \&$ Ing=pt\&tlng=pt 
23. Guillemin F. Cross-cultural adaptation and validation of health status measures. Scand J Rheumatol [Internet]. 1995 [cited 2016 Jan 31];24(2):61-3. Available from: https://doi. org/10.3109/03009749509099285

24. Pasquali L. Psicometria: teoria dos testes na Psicologia e na Educação. 5th ed. Petrópolis, RJ (BR): Editora Vozes; 2017.

25. Leplège A, Ecosse E, Verdier A, Perneger T. The French SF-36 Health Survey: translation, cultural adaptation and preliminary psychometric evaluation. J Clin Epidemiol. [Internet]. 1998 [cited 2016 Jan 30];51(11):1003-23. Available from: https://doi.org/10.1016/S0895-4356(98)00093-6 


\section{NOTES}

\section{ORIGIN OF THE ARTICLE}

Extracted from the Research - Knowledge about sexually transmitted infections, attitudes and embarrassment towards condom use in young university students, presented to the Pro-Rectory of Research and Graduate Studies of the Universidade de Rio Verde, Research Support Program, 2016.

\section{CONTRIBUTION OF AUTHORITY}

Study design: Moreira B.

Data collect: Moreira B, Rego UL.

Data analysis and interpretation: Moreira B, Rego UL, Patussi MP.

Discussion of the results: Moreira B, Rego UL, Souza SB.

Writing and/or critical review of the content: Moreira B, Pattusi MP.

Review and final approval of the final version: Moreira B, Pattussi MP.

\section{FUNDING INFORMATION}

Research Support Program of the Universidade de Rio Verde, Research Grant, internal call 01/2016 Dean of Research and Graduate Studies. Process Number: 007.2016.4.04.

\section{APPROVAL OF ETHICS COMMITTEE IN RESEARCH}

Approved by the Ethics Committee in Research with Human Beings of the Universidade de Rio Verde, opinion no.1,359,296/2015. CAAE: 50823915.1.0000.5077.

\section{CONFLICT OF INTEREST}

There is no conflict of interest.

\section{HISTORICAL}

Received: August 12, 2019.

Approved: March 18, 2020.

\section{CORRESPONDING AUTHOR}

Berenice Moreira

berenice@unirv.edu.br 